\title{
BIOÉTICA Y DISCAPACIDAD PSIQUIÁTRICA: ASPECTOS CLÍNICOS Y JURÍDICOS
}

\author{
Laura Rueda Castro* \\ María Angélica Sotomayor Saavedra**
}

Resumen: La presente monografía busca establecer un nexo interdisciplinario entre la bioética y los aspectos clínicos y jurídicos que se conectan en la atención, protección y rehabilitación de las personas afectadas por enfermedades psiquiátricas. El análisis se centra en aquellas personas adultas, privadas de razón como consecuencia de un proceso psicopatológico, titulares de todos sus derechos civiles, pero que, por motivos de la enfermedad, se encuentran con dificultades para ejercerlos.

Palabras clave: Bioética, enfermo psiquiátrico, incapacidad, demente

\section{BIOETHICS AN PSYCHIATRIC DISSABILITY: CLINICAL AN JURIDICAL APECTS}

Abstract: This monography seeks to establish an interdisciplinary link between bioethics and the clinical and juridical aspects that connect with attention, protection and rehabilitation of persons with psychiatric illness. The analysis is centered those adults, on deprived of reasoning because of a psycopathologic process, entitled to civil rights, but with dificulties to exercise them due to their illness.

Key words: Bioethics, psychiatric patient, incapacity, insane

\section{BIOETICA E INCAPACIDADE PSIQUIÁTRICA: ASPECTOS CLÍNICOS E JURÍDICOS}

Resumo: A presente monografia busca estabelecer nexo interdisciplinar entre bioética e aspectos clínicos e jurídicos na atenção, proteção e reabilitação de pessoas portadoras de enfermidades psiquiátricas. A análise é centrada em pacientes adultos privados de razão em decorrência de processo psicopatológico, titulares de todos direitos civis, mas que, em função da enfermidade, encontram-se em dificuldades para exercê-los.

Palabras chave: Bioética, paciente psiquiátrico, incapacidade, demente

Bioeticista, Terapeuta Ocupacional, Licenciada en Filosofía, Profesora Asistente, Facultad de Medicina Universidad de Chile. Correspondencia: ruedal@chi.ops-oms.org

** Abogada, Magíster (c) en Bioética, Asesora Jurídica, Facultad de Medicina Universidad de Chile.

Correspondencia: msotomay@canela.net.uchile.cl 
La bioética como disciplina emerge desde el estudio sistemático de los comportamientos humanos en el área de las ciencias de la vida y del cuidado de la salud. El presente trabajo se introduce en el análisis que puede desarrollar la bioética en el área específica de la psiquiatría y sus implicancias jurídicas. En este contexto es factible examinar problemáticas tales como confidencialidad de los tratamientos, uso de psicofármacos, investigación en psiquiatría, modos de internación involuntaria, participación de los pacientes en la toma de decisiones que los involucran, y muchas otras.

Las personas que desarrollan su quehacer profesional en psiquiatría, están conscientes de que, constantemente, deben transgredir la libertad de los pacientes para protegerlos de ellos mismos y de otras personas, como familiares o vecinos de los enfermos. Los estados de pérdida de juicio de la realidad y la falta de autocontrol de los impulsos despiertan en las personas que rodean al enfermo un gran temor. Por su parte, el equipo de salud encargado de la atención en las primeras etapas de la crisis mantiene una actitud de alerta y de prevención de riesgos.

El trabajo que presentamos busca un nexo interdisciplinario entre la bioética y los aspectos clínicos y jurídicos que se conectan en la atención, protección y rehabilitación de las personas afectadas por enfermedades psiquiátricas. Dada la amplitud del tema, nuestro análisis se centrará en aquellas personas adultas, privadas de razón como consecuencia de un proceso psicopatológico, titulares de todos sus derechos civiles, pero que, por motivo de la enfermedad, se encuentran con dificultades para ejercerlos.

Nos parece fundamental, en una primera instancia, expresar los argumentos que sustentan la aplicación de la bioética en la psiquiatría y el derecho. Respecto de la primera, los pa- cientes psiquiátricos -como todos los seres humanos- desarrollan capacidades en orden a satisfacer las necesidades básicas que le reportan un bienestar en su vida cotidiana ${ }^{1}$ : es lo que se denomina "lineamiento práctico". El tomar una decisión, por ejemplo, participa de este ámbito práctico porque implica que la persona asume roles y establece esquemas de actividades concordantes con dichos roles; ello se aprecia como rutinas cotidianas. Existe, además, lo que se denomina "lineamiento conceptual”, esto es, aquellos fundamentos que se encuentran o podrían encontrarse impresos detrás de las decisiones humanas; pertenecen también a este aspecto, ya que el hombre es parte de una situación macrosocial que lo obliga a asumir creencias, valores e ideologías que sustentan la elección de las decisiones. Éstos constituyen el esquema de usos sociales (morales) que cada persona debe cumplir para ser aceptada como miembro de la comunidad. El no cumplimiento de estos usos es sancionado por la sociedad, y las vías más habituales establecidas son las reclusiones psiquiátricas o penales, según corresponda.

Es necesario establecer los elementos morales que están presentes en una persona con capacidad de participar en una decisión, y apreciar en forma previa si una persona está en condiciones de participar socialmente con acierto. Los elementos morales que aparecen al tratar el tema son: el sentido moral, el buen juicio, la conciencia de cada situación, el aceptar límites, el mantener buenas costumbres. Para apreciar la expresión de los elementos morales se requiere determinar algunos comportamientos tipo que emite la persona que conserva su moralidad en forma habitual.

Para el desarrollo de este análisis es necesario el examen de los contenidos morales. Se

1 Max Neef M. Desarrollo a Escala Humana. Montevideo: Editorial Nordan - Comunidad; 1993: 49. 
trata, así, de un interés que se ajusta coherentemente a los propósitos de la bioética, ya que por constituir ésta el nexo entre dos planos de la vida humana se considera, precisamente, "la disciplina que combina el conocimiento biológico con el conocimiento de los sistemas de valores humanos" ${ }^{2}$. En esto se funda la idea de recuperar la identidad de personas morales en el ámbito de las enfermedades mentales.

En este sentido, la bioética, como disciplina sintetizadora de la ética filosófica, conserva el carácter especulativo y reflexivo de ésta, unido a los conocimientos teórico-prácticos que se requieren y se adquieren en el trabajo de las ciencias de la salud. La vida del ser humano necesita del cuidado de los elementos que la constituyen en su esencia, es decir, necesita salvaguardar sus valores para así conservar su propia humanidad, sin excepción de condiciones. La bioética asume esta tarea procurando guiar las acciones en la atención sanitaria sin que éstas dañen la integridad y dignidad de las personas involucradas. Se cuestiona dicha aplicación en dimensiones más específicas, como sectores de reclusión psiquiátrica. ¿Participan los pacientes psiquiátricos en las decisiones que los involucran? De no ser así, ¿es factible hablar de actos bioéticos generados única y exclusivamente desde posiciones directivas? En recintos psiquiátricos da la impresión de que no es así.

Después de varios años de práctica clínica en el área de la psiquiatría, escuchando, observando y compartiendo cotidianamente actividades con personas afectadas por la singular situación de ser enfermo mental, nos llama la atención que individuos con evidentes perturbaciones en los contenidos y curso formal del pensamiento, presenten comportamientos ade-

Potter V.R. Humility with Responsibility: A Bioethcs for Oncologists. Presidential Address. Cáncer Research 1975; 35: 2297- 9 . cuados en situaciones que surgen espontáneamente en el devenir diario. Ello les permite resolver en forma armónica hechos de bastante contingencia, como resolución de problemas o manejo de acontecimientos de emergencia.

En estas personas se percibe la vigencia de algo común a todos los seres humanos: existe el uso de las mismas palabras, se elaboran juicios, se comunican intenciones, se establecen conversaciones como una necesidad de formar vínculos. Lo anterior nos lleva a inferir que existe un sentimiento o sensación de humanidad que se conserva, una capacidad que aún persiste y que les permite distinguir lo bueno de lo malo, lo bello de lo feo, lo correcto de lo incorrecto.

\section{Ética y Práctica Psiquiátrica}

La capacidad humana que recogeremos para desarrollar la vinculación entre ética y actividad psíquica es la conocida bajo el concepto de "sentido común”, según la doctrina de Kant. Éste organiza la propiedad de juzgar que posee cada persona considerando la afectividad involucrada en las situaciones reales, junto con la base de conceptos teóricos que actúan a modo de principios fundamentales, como son las normas y reglas o juicios de valor.

La noción de "sentido común”, en general, puede ser definida como el efecto del juego de las capacidades mentales, en el supuesto de que opera bajo la disposición de las facultades que permiten cumplir una tarea específica. Se distinguen tres grandes facultades: el pensar, cuya dirección es el conocer, o sentido común lógico; la voluntad, cuya dirección es el desear hacer o sentido común moral; y el sentir, que guía la manifestación del placer o sentido común estético.

\footnotetext{
3 Kant I. Crítica de la Facultad de Juzgar. Buenos Aires: Editorial Losada S.A.; 1961.
} 
La persona con discapacidad psíquica, al igual que todo ser social, se encuentra inmersa en las normas culturales establecidas por la comunidad a la cual pertenece, conocidas como "buenas costumbres". Sin embargo, se ve a diario enfrentada a inclinaciones sensibles, también comunes a los otros individuos de la comunidad, como son el egoísmo, la vanidad, el miedo y la inseguridad, entre otras. Se suman a este ambiente relacional las alteraciones psicopatológicas propias de su condición particular, como son los trastornos en el enjuiciamiento de la realidad, la impulsividad, el egocentrismo, el desapego afectivo, etc.; alteraciones éstas que dificultan notablemente la interacción con otras personas.

Un examen de cada forma específica de “sentido común” nos muestra una vinculación con los tipos clínicos de desajustes conductuales que origina la enfermedad mental.

A. La alteración del sentido común lógico o el uso ilegítimo del sentido común lógico (de acuerdo con la doctrina de las facultades kantianas) se aprecia en aquellas personas que manifiestan a través de la expresión verbal, gestual y/o corporal, una percepción alterada del mundo que las rodea.

El juicio de la realidad se elabora a partir de la percepción del mundo externo, pero el ser humano también convive con su mundo interno de fantasías, imaginaciones y ensueños. Un individuo sano (con uso legal de sus facultades), delimita con precisión y claridad las fronteras que separan las realidades interna y externa. Logra aceptar una integración dialéctica entre lo real y lo irreal.

Cuando la irrealidad invade parcial o totalmente la realidad, la lógica del entendimiento habitual se subordina a la interpretación atípica que puede generar una persona. La falta de integración lógica entre ambas realidades se muestra en la escisión que se vive en la esquizofrenia.

Nuestro conocimiento del mundo y de nosotros mismos se expresa a través de las ideas. Ellas se constituyen desde una vivencia de la realidad para traducirse al final en un juicio de realidad. A partir de éste se construyen las ideas normales (lógicas).

Si la vivencia de la realidad es "correcta", el juicio de realidad será adecuado. Si la vivencia de realidad se perturba, el juicio de realidad será errado y las ideas que se construyan a partir de ese juicio serán desviadas de lo usual.

Las personas afectadas por esquizofrenia constituyen una gran población de enfermos mentales que, después de sufrir las fases agudas de la patología, van alcanzando lentamente la estabilización, pero sobre la base de un deterioro psicosocial, denominado defecto esquizofrénico. Este defecto no constituye un factor tan invalidante, como lo advierten familiares y personas dedicadas a su protección. En este grupo de personas centraremos más adelante nuestro análisis.

Para completar la interpretación de los otros cuadros psicopatológicos, a la luz de la descripción del sentido común, debemos completar el examen que hemos iniciado con las expresiones ilegítimas (morbosas) de las otras funciones del sentido común.

B. La alteración del sentido común moral (o su uso ilegítimo) provoca los desajustes conductuales más evidentes para la comunidad que convive con la persona afectada. Se traduce en la pérdida de los límites sociales, desadecuación del contexto cultural, falta de respeto u oposición activa a los usos sociales, y exagerado egocentrismo, entre otros rasgos. Estas características configuran el perfil de la persona definida 
clínicamente con un trastorno de personalidad. Ello se expresa técnicamente como un patrón permanente e inflexible de la experiencia interna y del comportamiento que se aparta acusadamente de las expectativas de la cultura y del ambiente sociocultural. Es estable a lo largo del tiempo y comporta malestar para el sujeto y su entorno.

La concordancia entre el entendimiento y la razón, como una actitud reflexiva para conocer las cosas sujetas a normas y aceptarlas, no está presente al desencajar esta función del sentido común. Según los criterios internacionales para el diagnóstico del trastorno antisocial, la persona con este problema presentaría:

- Fracaso para adaptarse a las normas sociales en lo que respecta al comportamiento legal, como lo indica el perpetrar repetidamente actos que son motivo de detención.

- Deshonestidad, expresada en la acción de mentir repetidamente, utilizar apodos, estafar a otros para obtener un beneficio personal o placer.

- Impulsividad o incapacidad para planificar el futuro.

- Irritabilidad y agresividad, indicadas por repetidos altercados verbales y físicos.

- Despreocupación imprudente por su seguridad o la de los demás.

- Irresponsabilidad persistente, indicada por la incapacidad de mantener un trabajo con constancia o de hacerse cargo de obligaciones económicas.

- Falta de remordimientos, como lo indica la indiferencia a la justificación de haber dañado o maltratado a otro.

Las alteraciones a este nivel de la conciencia moral resultan, por su propia definición, permanentes en el tiempo, y la atribución de responsabilidad a estos actos frente al entorno social, corresponde a la psiquiatría forense y sus implicancias penales.

C. La alteración del sentido común estético no provoca grandes conflictos en la convivencia social de los individuos. Tradicionalmente se acepta la particularidad en asuntos de gustos de cada persona. Se puede percibir su alteración cuando se presenta un desborde de la imaginación que no logra formularse en conceptos o expresiones entendibles para los demás, como es el caso del excentricismo y la bizarrería. Se trata de un sentido común que habitualmente permanece conservado, representando por ello una vía de comunicación, a veces más expedita que el diálogo formal para las personas con alteraciones en la expresión verbal lógica (por ejemplo: arteterapia, músicoterapia, aromaterapia).

La enfermedad mental representa un obstáculo importante que dificulta el desarrollo de lo que debe ser la vida humana, porque afecta, precisamente, la relación entre el individuo y el mundo. Sin embargo, las dificultades se van aminorando en la medida en que se alejan los períodos de crisis y se recibe atención especializada (psicofármacos, psicoterapia, rehabilitación).

Una patología psiquiátrica de larga evolución, que es origen de discapacidad psíquica permanente y que afecta a parte del grupo de personas recluidas en recintos psiquiátricos, es la esquizofrenia (alteración del sentido común lógico, preferentemente).

Las personas esquizofrénicas, en general, presentan dificultad en la comunicación con los demás, no utilizan del modo usual los comportamientos verbales y no verbales. Su mímica es pobre y sus gestos pueden parecer lentifica- 
dos, mecánicos y estereotipados. En algunos casos la expresión corporal es discordante con la emoción o relato verbal. Las categorías definidas internacionalmente clasifican los síntomas en positivos y negativos. Los primeros, que se presentan en brotes agudos de la enfermedad, corresponden a alteraciones en el contenido y en la forma de estructurar las ideas, alteraciones del lenguaje y comunicación y desorganización comportamental. Estos síntomas disminuyen en porcentaje importante con el tratamiento de psicofármacos ${ }^{4}$. Los síntomas negativos, producidos principalmente en etapas crónicas, son de intensidad variable y se relacionan directamente con el defecto esquizofrénico que provoca un considerable deterioro psicosocial: corresponden al aplanamiento afectivo, abulia, desgano. Existen antecedentes de que el aplanamiento afectivo produce una disminución en la expresión de emociones y lenguaje corporal, lo que genera un déficit en el desarrollo de habilidades comunicativas y, como consecuencia de lo mismo, en las habilidades sociales. Sin duda, esto interfiere en las relaciones interpersonales que puedan establecer las personas esquizofrénicas afectando, en forma primaria, las relaciones con su familia o personas cercanas. Además, es importante señalar el efecto que esto provoca en los demás, quienes perciben al esquizofrénico como desinteresado, retraído, callado.

Las manifestaciones anteriormente descritas varían en intensidad a medida que se com-

\footnotetext{
4 Los tratamientos psiquiátricos han sido beneficiados notablemente con el avance tecnológico a nivel de la farmacología. Las conductas agresivas e insociables han logrado modificarse con el uso de elementos químicos; ello ha significado el reintegro a la vida social, evitándose las reclusiones hospitalarias por largo tiempo. Sin embargo, el uso de los psicofármacos constituye un frecuente dilema bioético para los profesionales de la atención psiquiátrica pues todos presentan efectos secundarios, lo que requiere de un cuidadoso control de sus límites terapéuticos para no caer en casos de yatrogénesis.

Cf. López Azpitarte E. Ética y Vida: desafíos actuales. Cap. 16 “Los psi cofármacos”. Madrid: Ediciones Paulinas; 1991: 295-308.
}

pensan psicopatológicamente; así, las personas van recuperando su participación social y, al mismo tiempo, el ejercicio de sus derechos civiles. En este proceso debe participar el equipo interdisciplinario de salud que se desempeña en el área.

\section{Ética y Práctica Jurídica en Relación con la Discapacidad Psiquiátrica}

En esta parte del análisis abordaremos los elementos constitutivos del marco jurídico relativo a los distintos estados de incapacidad de las personas. Continuaremos con un acercamiento a los aspectos jurídicos que se relacionan con el sujeto portador de una enfermedad mental, en este caso esquizofrenia compensada y, finalmente, propondremos la integración de estos elementos en la atención y reeducación social del enfermo psiquiátrico.

Las diversas situaciones que afectan a los pacientes psiquiátricos plantean desafíos para el derecho que la ciencia jurídica suele enfrentar, a veces, sin mucha precisión. Desde luego, no se trata de un enfoque puramente jurídico, pues la ciencia del derecho se apoya también en la ciencia médica: es ésta la que determina -a veces también sin mucha precisión- los ámbitos de sanidad y enfermedad. Con todo, los efectos de tal determinación son conclusivos e importantes pues para el Derecho no existen estadios intermedios: el paciente psiquiátrico está o no privado de su capacidad para actuar en la vida jurídica.

Desde el punto de vista jurídico, la capacidad es la aptitud legal que tienen todos los individuos para adquirir derechos civiles y para ejercitarlos por sí mismos. Clásicamente se distingue entre capacidad de goce -la aptitud legal para adquirir derechos- y capacidad de ejercicio -la aptitud legal de una persona para ejercitar por sí misma los derechos que le competen, sin el ministerio o la autorización de otra-- La 
capacidad de goce la tiene todo individuo por el solo hecho de ser persona, siendo uno de los atributos esenciales de la personalidad. La capacidad de ejercicio, en cambio, si bien constituye la regla general, puede no estar presente en ciertas personas, las cuales, en atención a su edad, salud mental u otra causa expresamente contemplada por la ley, son consideradas incapaces de ejercer sus derechos. En otras palabras, si bien son titulares de derechos -es decir, poseen capacidad de goce- no pueden ejercerlos por sí mismas.

Las incapacidades de ejercicio pueden ser, básicamente, de dos tipos: absolutas y relativas. Son incapaces absolutos los dementes, los impúberes y los sordomudos que no pueden darse a entender por escrito. Son incapaces relativos los menores adultos y los pródigos o disipadores bajo interdicción de administrar sus bienes.

Las personas mentalmente sanas, en consecuencia, pueden encontrarse en una situación de incapacidad debido a su minoría de edad. En efecto, son incapaces absolutos los impúberes, es decir, el varón que no ha cumplido 14 años y la mujer que no ha cumplido 12 (artículo 26 del Código Civil). Estas personas, como -por lo demás- todos los incapaces absolutos, sólo pueden actuar en la vida jurídica representados. Por otra parte -y según hemos señalado más arriba- son incapaces relativos los menores adultos, esto es, las mujeres que han cumplido 12 años y los varones que han cumplido 14, pero que aún no han llegado a la mayoría de edad (18 años). Estas personas no carecen totalmente de juicio, como sí ocurre -según la ley- con los impúberes, pero no pueden administrar eficientemente sus negocios. En consecuencia, pueden actuar en la vida jurídica representados o autorizados por su representante legal.
En el ámbito de la bioética, esta incapacidad de los menores de edad tiende a ser morigerada. Así, existen diversos cuerpos normativos -especialmente instrumentos internacionales- que toman en consideración el asentimiento del niño, por ejemplo, para participar en una investigación científica, respecto de la cual los padres o los representantes legales del menor han otorgado su consentimiento. En este sentido, se otorga plena validez a la negativa del menor a participar en estas investigaciones, primando la voluntad del menor por sobre el consentimiento de los padres o representantes, cuando corresponda.

En el caso de individuos adultos, el ordenamiento jurídico chileno contempla tres casos de incapaces: los dementes, los sordomudos que no pueden darse a entender por escrito y los pródigos o disipadores bajo interdicción de administrar sus bienes. Los primeros, son incapaces absolutos porque carecen de voluntad jurídicamente eficaz; los segundos, por no poder exteriorizarla; los disipadores, por último -que son aquellos que dilapidan sus bienes en forma desproporcionada a sus haberes, manifestando una total falta de prudencia-son incapaces relativos, no son enfermos mentales y si tienen algún desequilibrio intelectual no es suficiente como para considerarlos absolutamente incapaces. La incapacidad de estos últimos, a diferencia de los dementes y sordomudos, se refiere sólo a los actos patrimoniales y no al derecho de familia; en consecuencia, podrán casarse, impugnar la paternidad que le ha sido reconocida, etc.

Los pródigos y los dementes que han sido puestos en entredicho de administrar sus bienes y los sordomudos que no pueden darse a entender por escrito requieren de un representante legal, que en este caso se denomina curador general (artículo 342 del Código Civil). Con todo, es del caso señalar que, en la 
práctica, estas reglas tienden a morigerarse, por cuanto existe una amplia gama de actos y contratos cotidianos, de cuantías menores, cuya celebración no requiere el cumplimiento de solemnidades especiales -se trata de contratos consensuales, en su gran mayoría- que estos incapaces suelen celebrar sin que intervenga para nada su representante legal (por ejemplo, contrato de transporte y compraventa de especies muebles de menor valor, entre otros).

Ahora bien, la ley establece que toda persona es legalmente capaz, excepto aquellas que la ley declare incapaces (artículo 1447 del Código Civil); en consecuencia, la capacidad se presume $\mathrm{y}$, por consiguiente, debe probar la incapacidad el que la alegue.

En la materia que nos ocupa, es del caso señalar que, para que una persona se encuentre en interdicción por demencia, es necesario que el juez decrete judicialmente que el curador está autorizado para ejercer su cargo.

La denominación que utiliza el legislador civil chileno para referirse a esta clase de incapaces absolutos no debe entenderse en el sentido que la psiquiatría moderna da a la expresión demente, sino que alusiva a todo aquel que esté privado de razón o que tenga sus facultades mentales substancialmente alteradas, es decir, como sinónimo de enfermedad mental (así, por ejemplo, el artículo 456 del Código Civil dice que el adulto que se halla en un estado habitual de demencia, deberá ser privado de la administración de sus bienes, aunque tenga intervalos lúcidos).

En materia penal, la capacidad equivale a la imputabilidad y la incapacidad se traduce en inimputabilidad. A este respecto, el ordenamiento jurídico chileno establece que está exento de responsabilidad penal "el loco o demente, a no ser que haya obrado en un intervalo lúcido (...)” (artículo 10 del Código Penal). Al igual que en el caso de la legislación civil, las expresiones “loco o demente” no están empleadas en un sentido técnico.

Respecto de los enfermos hospitalizados en establecimientos de atención psiquiátrica, el Código Sanitario (artículo 133) establece que los directores de estos establecimientos serán curadores provisorios de los bienes de dichos enfermos, cuando carecieren de curador o no estén sometidos a patria potestad, mientras permanezcan internados o no se les designe curador, de acuerdo con las normas del derecho común.

En nuestra cultura judeocristiana, en una primera etapa, los enfermos mentales graves fueron considerados como "castigo de Dios", “figuras demoníacas” o “encarnaciones del demonio", con el consiguiente tratamiento de crueldad y reclusión de por vida. Con posterioridad, el enfermo mental se “domestica” mejorando el trato a su respecto. Es ésta la época del desarrollo de las ciencias del comportamiento, que buscan las causas del estado patológico y consideran la posibilidad de reeducación. La etapa en que nos encontramos actualmente se caracteriza por la reivindicación de los derechos de las personas, independientemente de las diversas y específicas situaciones en que éstas puedan encontrarse. Así, surgen los catálogos de derechos de los consumidores, de los pacientes, de los enfermos mentales, de los impedidos, entre otros. En este mismo sentido, diversos instrumentos internacionales han establecido estadios intermedios entre capacidad e incapacidad civil, empleando la denominación "impedido", reconociendo derechos y estableciendo cautelas y obligaciones de la sociedad en relación con ellos.

La Declaración de los Derechos de los Impedidos, proclamada por la Asamblea General de las Naciones Unidas, en su resolución 3447, de 1975, señala que el término "impedido" designa a toda persona incapacitada de subvenir 
por sí misma, en su totalidad o en parte, a las necesidades de una vida individual o social normal a consecuencia de una deficiencia congénita o no, de sus facultades físicas o mentales.

Agrega esta Declaración que el impedido tiene esencialmente derecho a que se respete su dignidad humana y que tiene los mismos derechos fundamentales que sus conciudadanos de la misma edad, lo que supone, en primer lugar, el derecho a disfrutar de una vida decorosa lo más normal y plena posible. Asimismo, hace aplicable a los impedidos el párrafo 7 de la Declaración de los Derechos del Retrasado Mental -proclamada por la Asamblea General de las Naciones Unidas en su resolución 2856, de 20 de diciembre de 1971-el cual prescribe que, si debido a la gravedad de su impedimento, no es capaz de ejercer efectivamente todos sus derechos, o si se hace necesario limitar o incluso suprimir tales derechos, el procedimiento que se emplee a los fines de esa limitación o supresión deberá entrañar salvaguardas jurídicas que protejan al impedido de abuso. Dicho procedimiento deberá basarse en una evaluación de su capacidad social realizada por expertos calificados. Asimismo, tal limitación o supresión quedará sujeta a revisiones periódicas y reconocerá el derecho de apelación a autoridades superiores.

El ordenamiento jurídico chileno, en la Ley № 19.284 de 1994 -que establece normas para la plena integración social de personas con discapacidad- señala en su artículo $3^{\circ}$ que “(...) se considera persona con discapacidad a toda aquella que, como consecuencia de una o más deficiencias físicas, síquicas o sensoriales, congénitas o adquiridas, previsiblemente de carácter permanente y con independencia de la causa que las hubiere originado, vea obstaculizada, en a lo menos un tercio, su capacidad educativa, laboral o de integración social". Por su parte, el Reglamento para la Evaluación y
Calificación de la Discapacidad (D.S. N 2.505, del Ministerio de Salud, de 1995), define en el artículo $2^{\circ}$ lo que se entiende por discapacidad educativa, laboral y de integración social. Con respecto a esta última, señala que "es aquella en la que una persona por sus deficiencias psíquica o mental, física y/o sensorial presenta un menoscabo de su capacidad de inserción en las actividades propias de la sociedad humana, de la familia y/o de los grupos organizados de la sociedad, viendo disminuidas así sus posibilidades para realizarse material y espiritualmente en relación a una persona no discapacitada en situación análoga de edad, sexo, formación, capacitación, condición social y familiar y de igual localidad geográfica”.

El derecho, en general, no hace mayores diferenciaciones en relación con los pacientes psiquiátricos, por cuanto no discrimina según los procesos patológicos de las enfermedades mentales, ni respecto de la conservación de las diversas formas de sentido a que se ha aludido más arriba. Estimamos, en consecuencia, que aquí se abre un gran espacio para la bioética, en la consideración de la autonomía moral, con base en el respeto a la dignidad humana. Bajo esta mirada, hechos jurídicamente aceptables, pueden no serlo desde el punto de vista ético, como, por ejemplo, disponer de los bienes del pupilo-paciente sin recibir su asentimiento, disponer el cambio de su lugar de residencia u otros análogos.

El enfoque del mejor interés del incapaz debiera orientar a las personas a su cargo. Jurídicamente está consagrado en el Código Civil chileno respecto del menor e, implícitamente, respecto de las personas sujetas a guarda, quienes, al decir de la ley, deben dar a los incapaces la protección debida en interés de ellos (artículos 338 y siguientes del Código Civil). Dicha visión permite considerar también la opinión del sujeto, en la medida que se concilie con su mejor interés. 
Desde la bioética, una ética de la fragilidad nos parece pertinente, ya que implica el deber moral de proteger a los más débiles. Precisamente, ésta ha sido la historia de los derechos humanos, una reacción frente al abuso de que han sido víctimas los más débiles. La protección de tales derechos representa un claro avance ético de la humanidad.

La fragilidad del impedido puede ser violentada por actos de comisión o de omisión. En el primer caso podría estar, por ejemplo, el sometimiento del incapaz mental a investigaciones que no supongan un beneficio real y directo para su salud o bien que no consideren su rechazo. Ello a menos que, cumpliéndose las restantes condiciones de resguardo que se establecen para personas sanas, el experimento tenga por objeto -mediante una mejora significativa del conocimiento científico del estado de la persona, de su enfermedad o de su trastorno- contribuir a lograr en un determinado plazo, resultados que permitan obtener un beneficio para la persona afectada o para otras personas de la misma categoría de edad o que padezcan la misma enfermedad, el mismo trastorno o las mismas características. Lo anterior, siempre y cuando el experimento sólo presente para la persona un riesgo o inconveniente mínimo 5 .

Finalmente, otra forma de violencia, esta vez por omisión, se produce frente al abandono del impedido por la sociedad, comunidad, equipo sanitario o familia, quienes tienen como imperativo ético y jurídico, la obligación de brindarles protección.

Las medidas de protección, como se señaló, están establecidas en el mejor interés del impedido y no pueden significar, en modo alguno,

Convenio para la Protección de los Derechos Humanos y de la Dignidad del Ser Humano con respecto a las aplicaciones de la Biología y de la Medicina: Convenio sobre los Derechos Humanos y la Biomedicina. Consejo de Europa, 4 de abril de 1997. Revista de Derecho y Genoma Humano 1997; 7. formas de conculcar los derechos que a éste competen. Habrá situaciones en que, respecto de determinados estadios de enfermedades psiquiátricas, será indispensable aplicar medidas de contención u otras que impliquen limitación de la libertad, como, por ejemplo, internación obligatoria en establecimientos psiquiátricos. Si así fuere, éstas debieran circunscribirse al tiempo indispensable para adoptar las medidas médicas que el impedido requiera, protegiendo también, y en subsidio, a la comunidad.

En este sentido, y conforme a lo ya expresado, podemos concluir que:

- El derecho sólo toca a las situaciones en que están en riesgo los derechos de las personas, momento en el cual la norma jurídica o ley establece salvaguardas. En lo no reglado, está el amplio espacio de libertad, tanto respecto del paciente como de la familia u organización social que la reemplaza.

- La psiquiatría, en especial cuando sus acciones están destinadas a la manutención o recuperación de la capacidad autogestora de las personas, enfrenta la necesidad imperiosa de definir conceptos y normas que regulen éticamente las relaciones entre los miembros del equipo de salud y las personas afectadas por discapacidad psíquica.

- En la base de toda norma que pueda establecerse está el respeto de la dignidad humana, lo que implica que cualquiera sea la naturaleza o gravedad de los trastornos o deficiencias que el impedido (en este caso mental) sufra, tiene los mismos derechos fundamentales que el resto de las personas de su edad. Ello implica también el derecho a disfrutar de una vida decorosa, lo más normal y plena posible. 


\section{Bibliografía}

Allport G. La Personalidad. Su configuración y desarrollo. Barcelona: Editorial Herder; 1973.

American Psychological Association. Code of Professional Ethics. Washington DC: Am.Psy.Ass.; 1987.

American Psychiatric Association. Model Law on Civil Commitment of the Mentally ill. En: APA Issues in Forensic Psychiatry. Washington DC: Am.Psy.Press; 1984.

Benzi I. El Sentido Común y la Comunicabilidad Universal en Kant. Santiago de Chile: Publicaciones Especiales de la Universidad de Chile. Serie Ensayos 2ª ed.: 1981.

Benzi I. Razón y Libertad. Capacidades humanas de cumplir lo humano. Santiago de Chile: Publicaciones Especiales de la Universidad de Chile. Serie Ensayos; 1987.

Capponi R. Psicopatología y Semiología Psiquiátrica. Santiago de Chile: Editorial Universitaria; 1987.

Consejo de Europa. Convenio para la Protección de los Derechos Humanos y la Dignidad del Ser Humano con respecto a las aplicaciones de la Biología y la Medicina. Revista de Derecho y Genoma Humano 1997; 7.

Franca-Tarragó O. Ética para Psicólogos: Introducción a la Psicoética. Bilbao: Editorial Descleé de Brouwer; 1996.

Gafo J. 10 Palabras Clave en Bioética. Navarra: Verbo Divino; 1993.

Gracia D. Fundamentos de Bioética. Madrid: Eudema S.A.: 1989.

Gracia D. Introducción a la Bioética: Siete Ensayos. Bogotá: Editorial El Búho; 1991.

Gracia D. Bioética Clínica. Ética y Vida. Bogotá: Editorial El Búho; 1998.

Gracia D. Ética en los Confines de la Vida. Bogotá: Editorial El Búho: 1998.

Hooft PF. Bioética y Derechos Humanos. Buenos Aires: Ediciones Desalma; 1999.

Kant E. Crítica de la Facultad de Juzgar, Buenos Aires: Editorial Losada S.A.; 1961.

López Azpitarte E. Ética y Vida: desafíos actuales. Madrid: Ediciones Paulinas; 1991.

Max-Neef M. Desarrollo a Escala Humana. Barcelona: Icaria Editorial; 1994.

Pacheco M. Derechos Humanos. Documentos Básicos. Santiago de Chile: Editorial Jurídica de Chile; 1992.

Rosenbaum M. Ética y Valores en Psicoterapia. México: Fondo de Cultura Económica; 1985.

Rueda L. Conciencia Moral en las personas con discapacidad de causa psíquica. Santiago de Chile: Programa Regional de Bioética OPS/OMS; 1999. 